
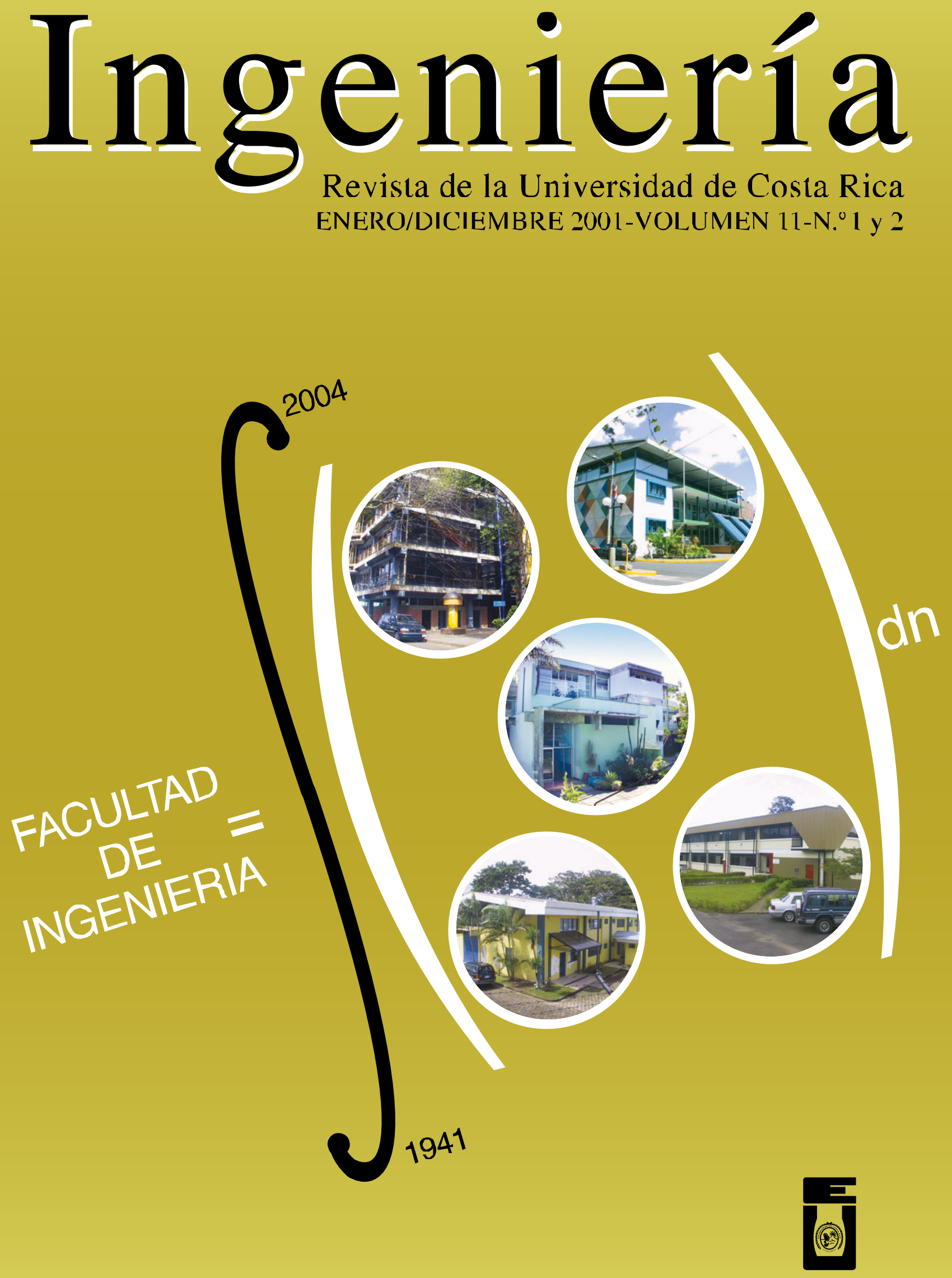


\title{
PROPIEDADES Y CAPACIDADES DE LOS $78 \% 6$ ESTRUCTURALES LAMINADOS EN FRÍO Y PRODUCIDOS EN COSTA RICA
}

Marija Romanjek

\section{Resumen}

En este artículo se presentan los resultados de los cálculos hechos por la autora con el propósito de facilitar los diseños de las estructuras hechas en tubos estructurales. Los cálculos se realizaron para todos las secciones tipo "tubo estructural" rectangular que se producen actualmente en Costa Rica por las empresas METALCO y TUBOTICO, considerando las características del acero utilizado. Se presentan tablas con las propiedades geométricas de las secciones de los perfiles calculadas con base en las dimensiones declaradas por los fabricantes, el límite de fluencia incrementado en compresión y en flexión, las capacidades nominales de los perfiles para la flexión y cortante, capacidades de las vigas con flexión y cortante combinados para el método de esfuerzos de trabajo (ASD) y para el método de esfuerzos últimos (LRFD), la fuerza crítica nominal para el pandeo del alma bajo una fuerza concentrada y las capacidades nominales de los perfiles considerados como columnas. Las tablas y sus datos cumplen con las especificaciones de American Iron and Steel Institute (AISI) y son de utilidad para los diseñadores de los sistemas estructurales, debido a que actualmente no hay manuales confiables en el país para tal efecto.

\begin{abstract}
This article presents the results of the calculations made by the author with the purpose of facilitating the design of structures made with square structural tubing. The calculations were made for all the sections "square structural tubing" that are nowadays produced by METALCO and TUBOTICO, considering the characteristics of the steel used. There are presented also, charts with the geometric properties of the sections of the members calculated on base with the measures declared by the producers, the yield point incremented for compression, and for bending yield point, capacities of the beams on bending and shear, capacities of the beams on combined bending and shear for the method of Allowable Stress Design (ASD), and for the Load and Resistance Factor Design (LRFD), and the nominal web crippling strength, and nominal axial strength. The charts and their data are according to the specifications of the American Iron and Steel Institute (AISI) and are of utility for the designers of structu-ral systems, considering that there are no trusting manuals in the country for that purpose.
\end{abstract}

\section{TUBO ESTRUCTURAL PRODUCIDO EN COSTA RICA}

\section{PROPIEDADES GEOMÉTRICAS DE LAS SECCIONES}

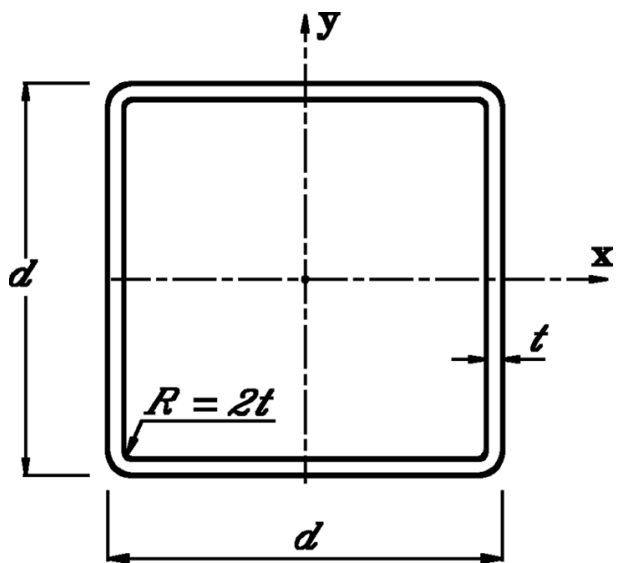

Figura \#1 
Tabla \#1 Propiedades geométricas de las secciones calculadas basándose en las dimensiones indicadas por los fabricantes.

\begin{tabular}{|c|c|c|c|c|c|c|c|}
\hline & \multirow[t]{2}{*}{ SECCIÓN } & d & $t$ & A & $I_{x}=I_{y}$ & $S_{x}=S_{y}$ & $r_{x}=r_{y}$ \\
\hline & & $\mathrm{cm}$ & $\mathrm{cm}$ & $\mathrm{cm}^{2}$ & $\mathrm{~cm}^{4}$ & $\mathrm{~cm}^{3}$ & $\mathrm{~cm}$ \\
\hline \multirow{7}{*}{$\begin{array}{l}M \\
E \\
T \\
A \\
L \\
C \\
O\end{array}$} & $72 \times 72 \times 1.2$ & 7,2 & 0,12 & 3,34 & 27,6 & 7,67 & 2,88 \\
\hline & $72 \times 72 \times 1.5$ & 7,2 & 0,15 & 4,13 & 33,8 & 9,39 & 2,86 \\
\hline & $72 \times 72 \times 1.9$ & 7,2 & 0,19 & 5,17 & 41,6 & 11,57 & 2,84 \\
\hline & $92 \times 92 \times 1.2$ & 9,2 & 0,12 & 4,30 & 58,6 & 12,74 & 3,69 \\
\hline & $92 \times 92 \times 1.5$ & 9,2 & 0,15 & 5,33 & 72,1 & 15,67 & 3,68 \\
\hline & $92 \times 92 \times 1.9$ & 9,2 & 0,19 & 6,69 & 89,4 & 19,43 & 3,65 \\
\hline & & & & & & & \\
\hline \multirow[b]{3}{*}{$\mathrm{T}$} & $38 \times 38 \times 2.4$ & 3,8 & 0,238 & 3,15 & 6,32 & 3,33 & 1,417 \\
\hline & $50 \times 50 \times 1.8$ & 5,0 & 0,180 & 3,33 & 12,58 & 5,03 & 1,943 \\
\hline & $50 \times 50 \times 2.4$ & 5,0 & 0,238 & 4,29 & 15,65 & 6,26 & 1,910 \\
\hline$U$ & $72 \times 72 \times 1.8$ & 7,2 & 0,180 & 4,92 & 39,7 & 11,04 & 2,84 \\
\hline B & $72 \times 72 \times 3.2$ & 7,2 & 0,317 & 8,30 & 63,4 & 17,62 & 2,77 \\
\hline 0 & $100 \times 100 \times 1.8$ & 10,0 & 0,180 & 6,93 & 110,2 & 22,0 & 3,99 \\
\hline $\mathrm{T}$ & $100 \times 100 \times 3.2$ & 10,0 & 0,317 & 11,85 & 181,2 & 36,2 & 3,91 \\
\hline 1 & $125 \times 125 \times 1.8$ & 12,5 & 0,180 & 8,73 & 219 & 35,0 & 5,01 \\
\hline C & $125 \times 125 \times 3.2$ & 12,5 & 0,317 & 15,02 & 365 & 58,5 & 4,93 \\
\hline \multirow[t]{4}{*}{0} & $125 \times 125 \times 4.8$ & 12,5 & 0,475 & 21,9 & 513 & 82,2 & 4,84 \\
\hline & $150 \times 150 \times 1.8$ & 15,0 & 0,180 & 10,53 & 383 & 51,0 & 6,03 \\
\hline & $150 \times 150 \times 3.2$ & 15,0 & 0,317 & 18,19 & 645 & 86,0 & 5,95 \\
\hline & $150 \times 150 \times 4.8$ & 15,0 & 0,475 & 26,6 & 917 & 122,2 & 5,87 \\
\hline
\end{tabular}

Donde:

$A=\quad$ área de la sección

$I_{x}, I_{y}=$ momentos de inercia de la sección con respecto a los ejes $x$ y $y$, respectivamente $S_{x}, S_{y}=$ módulos elásticos de la sección con respecto a los ejes $x$ y $y$, respectivamente $r_{x}, r_{y},=$ radios del giro de la sección con respecto a los ejes $x$, y $y$ respectivamente 
Límite de Fluencia Mínimo del Acero Virgen: $F_{y}=227 \mathrm{MPa}$

Esfuerzo Último Mínimo del Acero Virgen:

$F_{u}=340 \mathrm{MPa}$

Módulo de Elasticidad: $E=203 \mathrm{GPa}$

Módulo de Rigidez: $G=78 \mathrm{GPa}$

Tabla \#2 Límite de Fluencia Incrementado, Fya, según la Sección A7.2 de Cold Formed Steel Design Manual, edit. AISI, con Fyf $=227 \mathrm{MPa}$

\begin{tabular}{|c|c|c|c|}
\hline & SECCIÓN & COMPRESIÓN & FLEXIÓN \\
\hline & & $\mathrm{MPa}$ & $\mathrm{MPa}$ \\
\hline \multirow{7}{*}{$\begin{array}{l}M \\
\text { E } \\
T \\
A \\
L \\
C \\
O\end{array}$} & $72 \times 72 \times 1.2$ & $237^{*}$ & $245^{*}$ \\
\hline & $72 \times 72 \times 1.5$ & $239^{*}$ & $250^{*}$ \\
\hline & $72 \times 72 \times 1.9$ & 243 & 255 \\
\hline & $92 \times 92 \times 1.2$ & $235^{*}$ & $245^{*}$ \\
\hline & $92 \times 92 \times 1.5$ & $236^{*}$ & $250^{*}$ \\
\hline & $92 \times 92 \times 1.9$ & $239^{*}$ & $255^{*}$ \\
\hline & & & \\
\hline \multirow{13}{*}{$\begin{array}{l}\mathrm{T} \\
\mathrm{U} \\
\mathrm{B} \\
\mathrm{O} \\
\mathrm{T} \\
\mathrm{I} \\
\mathrm{C} \\
\mathrm{O}\end{array}$} & $38 \times 38 \times 2.4$ & 267 & 290 \\
\hline & $50 \times 50 \times 1.8$ & 249 & 265 \\
\hline & $50 \times 50 \times 2.4$ & 257 & 276 \\
\hline & $72 \times 72 \times 1.8$ & 242 & 254 \\
\hline & $72 \times 72 \times 3.2$ & 254 & 273 \\
\hline & $100 \times 100 \times 1.8$ & $238^{*}$ & $247^{*}$ \\
\hline & $100 \times 100 \times 3.2$ & 246 & 261 \\
\hline & $125 \times 125 \times 1.8$ & $235^{*}$ & $243^{*}$ \\
\hline & $125 \times 125 \times 3.2$ & 242 & 254 \\
\hline & $125 \times 125 \times 4.8$ & 250 & 267 \\
\hline & $150 \times 150 \times 1.8$ & $234^{*}$ & $240^{*}$ \\
\hline & $150 \times 150 \times 3.2$ & $239^{*}$ & $250^{*}$ \\
\hline & $150 \times 150 \times 4.8$ & 246 & 261 \\
\hline
\end{tabular}

* No se debe usar debido a que la sección no es completamente efectiva y el factor de reducción $\rho$ es menor de uno. Usar $F_{y}=227 \mathrm{MPa}$ 
Tabla \#3 Capacidad Nominal en Tracción, $T_{n}$ (según la Sección C2 de AISI)

CAPACIDADES DE LOS TUBOS ESTRUCTURALES

En los cálculos de las capacidades se usaron los esfuerzos de fluencia incrementados por el trabajo en frío, según lo permiten las normas de American Iron and Steel Institute (AISI)

Donde: $d=$ peralte de la sección

$t=$ espesor de los perfiles

$A=$ área de la sección

$F_{y a}=$ límite de fluencia incrementado para la tracción

$T_{n}=$ tracción nominal, debe modificarse con el Factor de Seguridad, $\Omega_{\mathrm{t}}=1,67$ o con el Factor de Resistencia, $\Phi_{\mathrm{t}}=0,95$, según el método de diseño que se está usando. 
Tabla \#4 Capacidad Nominal para la flexión (C3, AISI)

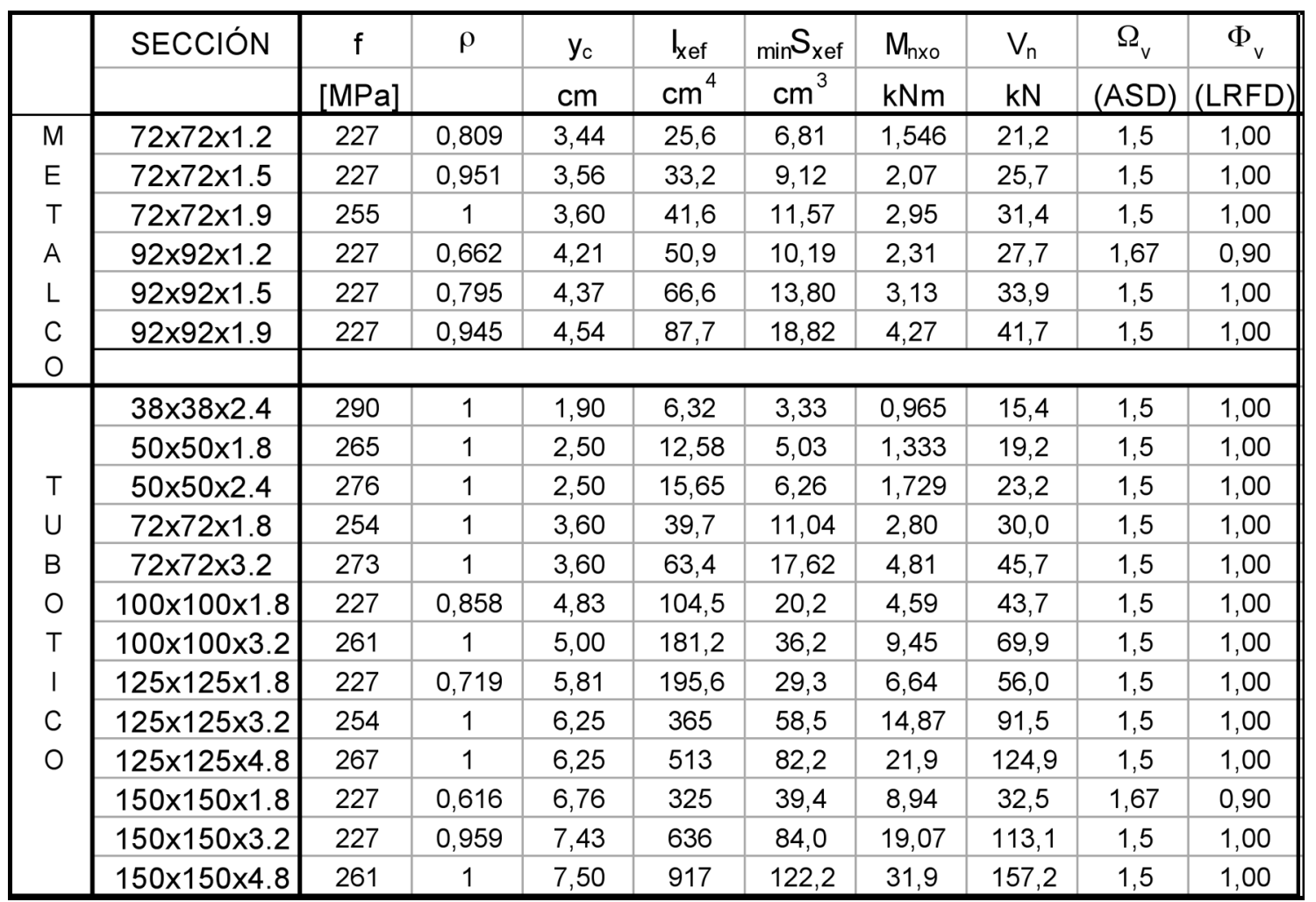

Donde:

$f=$ esfuerzo máximo en la sección (límite de fluencia incrementado para la flexión donde se permite o límite de fluencia del acero virgen).

$I_{x e f}=$ momento de inercia de la sección efectiva con respecto al eje $x$.

$M_{n x o}=$ momento nominal de la sección para la flexión alrededor del eje $x$ centroidal con el esfuerzo máximo $f$. Debe modificarse con el Factor de Seguridad o con el Factor de Resistencia.

- usando el Método de Esfuerzos de Trabajo (ASD) se divide con el Factor de Seguridad: $\Omega_{\mathrm{b}}=, .67$

- usando el Método de Esfuerzos Últimos (LRFD) se multiplica con el Factor de Resistencia: $\Phi_{\mathrm{b}}=0,95$ $\min S_{x e f}=$ módulo mínimo efectivo de la sección para la flexión alrededor del eje $x$.

$V_{n}=$ Fuerza Cortante Nominal $\left(\operatorname{con} F_{y}=227\right.$ $\mathrm{MPa}$ ). Debe modificarse con el Factor de Seguridad $\Omega_{\mathrm{v}}$ o con el Factor de Resistencia $\Phi_{\mathrm{v}}$, indicados en la Tabla 4 y según el método de diseño que se esté usando.

$y_{c}=$ posición del eje neutro, eje $\mathrm{x}$.

$\rho$ = factor de reducción de la sección.

NOTA: Este tipo de secciones no son susceptibles a pandeos lateral o torsional y por lo mismo las tablas no contienen los valores de las distancias máximas entre arriostres, $L_{u}$. 
Tabla \#5 Capacidades de las Vigas con Flexión y Cortante Combinados MÉTODO DE ESFUERZOS DE TRABAJO (ASD) (C3.3.1, AISI)

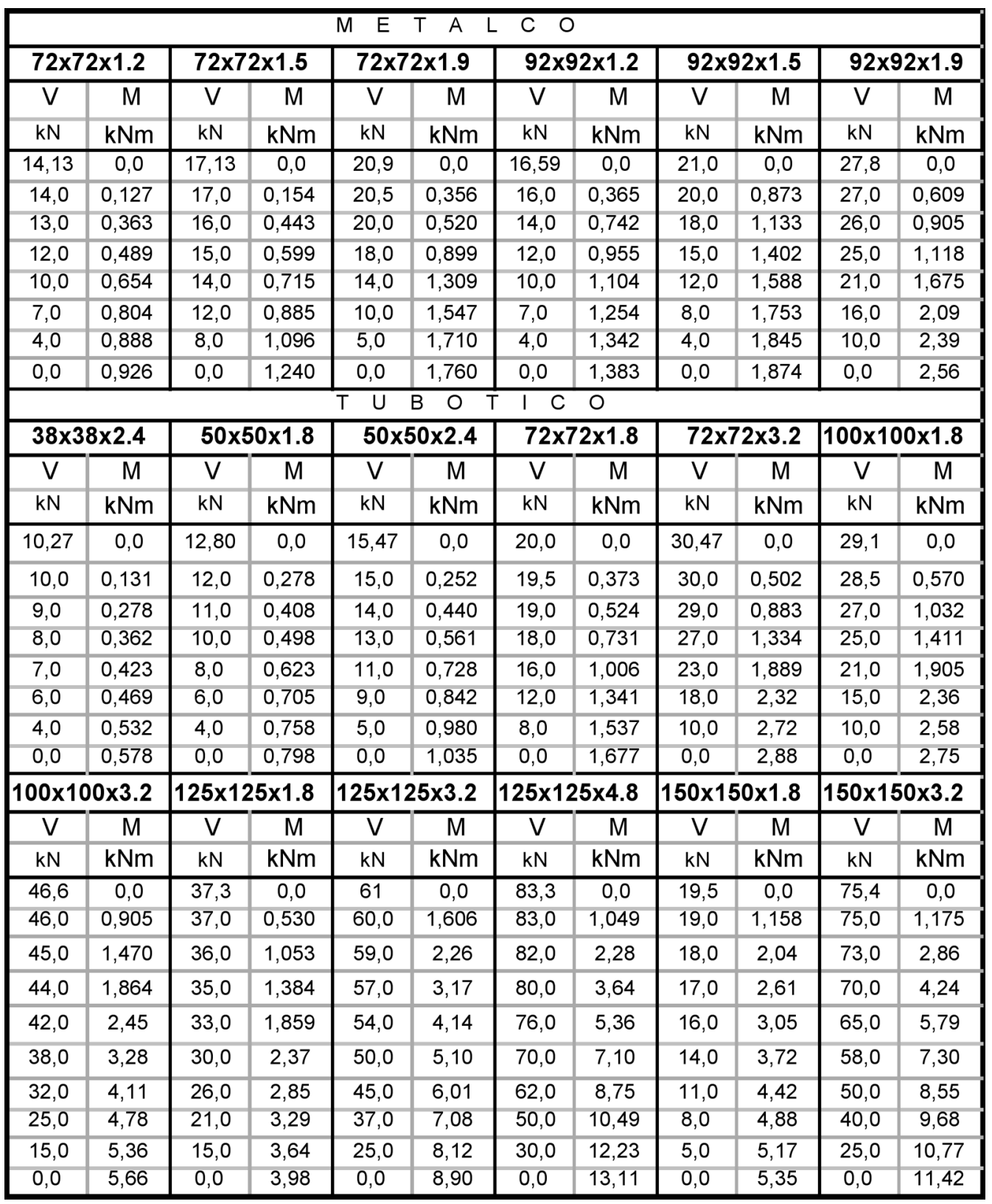


TABLA \#5 continuación

\begin{tabular}{|c|c||}
\hline \multicolumn{2}{|c|}{ TUBOTICO } \\
\hline $\mathbf{1 5 0 \times 1 5 0 \times 4 . 8}$ \\
\hline $\mathrm{V}$ & $\mathrm{M}$ \\
\hline $\mathrm{kN}$ & $\mathrm{kNm}$ \\
\hline 104,8 & 0,0 \\
\hline 104,0 & 2,36 \\
\hline 102,0 & 4,39 \\
\hline 98,0 & 6,77 \\
\hline 92,0 & 9,15 \\
\hline 83,0 & 11,66 \\
\hline 71,0 & 14,05 \\
\hline 52,0 & 16,58 \\
\hline 30,0 & 18,30 \\
\hline 0,0 & 19,10 \\
\hline
\end{tabular}

\section{MÉTODO DE ESFUERZOS DE TRABAJO \\ (ASD) (C3.3.1, AISI)}

Donde:

$V=$ fuerza Cortante en la Sección del Perfil.

$M=$ momento Permisible en la Sección del Perfil para la flexión alrededor del eje $x$ en la presencia de la fuerza cortante $V$.

NOTA: La Tabla 5 no se aplica en el caso de la combinación de flexión y fuerza concentrada (pandeo del alma) sea ésta la carga o la reacción. En este caso se debe satisfacer la siguiente ecuación de interacción (ver Sección C3.5 de AISI):

Para un alma simple y no reforzada
$1.2\left(\frac{\Omega_{w} P}{P_{n}}\right)+\left(\frac{\Omega_{b} M}{M_{n x o}}\right) \leq 1.5$

[Ec. C3.5.1-1]

Donde:

$P=$ fuerza concentrada en la presencia del momento.

$M=$ momento en la sección ó en la sección muy cercana al punto de aplicación del $P$.

$P_{n}=$ fuerza nominal del pandeo del alma en ausencia del momento (ver Tabla 7)

$M_{n x o}=$ momento nominal alrededor del eje $x$ centroidal (ver Tabla 4) 
Tabla \#6 Capacidades de las Vigas con Flexión y Cortante Combinados MÉTODO DE ESFUERZOS ÚLTIMOS (LRFD) (C3.3.2, AISI)

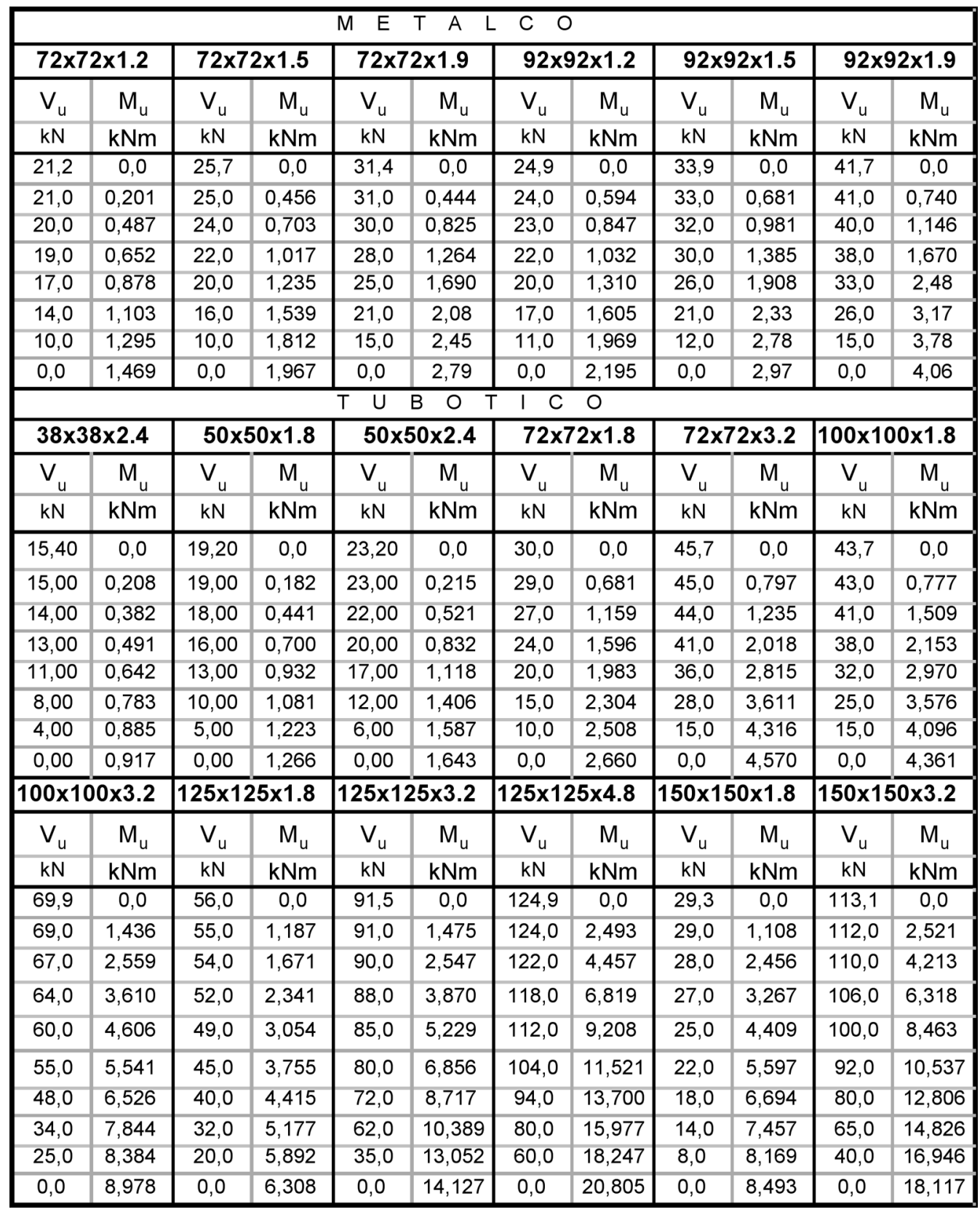


TABLA \#6 continuación

\begin{tabular}{|c|c||}
\hline \multicolumn{2}{|c|}{ TUBOTICO } \\
\hline $\mathbf{1 5 0 \times 1 5 0 \times 4 . 8}$ \\
\hline $\mathrm{V}_{\mathrm{u}}$ & $\mathrm{M}_{\mathrm{u}}$ \\
\hline $\mathrm{kN}$ & $\mathrm{kNm}$ \\
\hline 157,9 & 0,0 \\
\hline 156,0 & 3,74 \\
\hline 153,0 & 6,96 \\
\hline 148,0 & 10,22 \\
\hline 140,0 & 13,78 \\
\hline 130,0 & 17,04 \\
\hline 110,0 & 21,6 \\
\hline 85,0 & 25,5 \\
\hline 50,0 & 28,7 \\
\hline 0,0 & 30,3 \\
\hline
\end{tabular}

Donde

$V_{u}=$ fuerza cortante en la sección.

$M_{u}=$ momento permisible en la sección en la presencia de la fuerza cortante $V_{u}$.

NOTA: La Tabla 6 no se aplica en el caso de la combinación de flexión y fuerza concentrada (pandeo del alma) sea ésta la carga o la reacción. En este caso se debe satisfacer la siguiente ecuación de interacción (ver Sección C3.5 de AISI):

Para un alma simple y no reforzada:

$1.07\left(\frac{P_{u}}{\Phi_{w} P_{n}}\right)+\left(\frac{M_{u}}{\Phi_{b} M_{n x o}}\right) \leq 1.42$

[Ec. C3.5.2-1]
Donde:

$P_{u}=$ fuerza concentrada en la presencia del momento.

$M_{u}=$ momento en la sección o en la sección muy cercana al punto de aplicación del $P_{u}$.

$P_{n}=$ fuerza nominal del pandeo del alma en ausencia del momento (ver Tabla 7).

$M_{n x o}=$ momento nominal alrededor del eje $x$ centroidal (ver Tabla 4).

$\Phi_{\mathrm{b}}=$ coeficiente de resistencia para la flexión = 0.95

$\Phi_{\mathrm{w}}=$ coeficiente de resistencia para el pandeo 
PANDEO DEL ALMA DEBIDO A UNA FUERZA CONCENTRADA

Según la Sección C3.4 de AISI. Calculado con $F_{y}=227 \mathrm{MPa}$.

Tabla \#7a Fuerza Crítica Nominal del Pandeo del Alma,

$P_{n}$, para los productos de METALCO

\begin{tabular}{|c|c|c|c|c|}
\hline SECCION & $\begin{array}{c}\text { CASO A } \\
\text { Pn } \\
(\mathrm{kN})\end{array}$ & $\begin{array}{c}\text { CASO B } \\
\text { Pn } \\
(\mathrm{kN})\end{array}$ & $\begin{array}{c}\text { CASO C } \\
\text { Pn } \\
(\mathrm{kN})\end{array}$ & $\begin{array}{c}\text { CASO D } \\
\text { Pn } \\
(\mathrm{kN})\end{array}$ \\
\hline \multicolumn{5}{|l|}{$\mathrm{N}=50 \mathrm{~mm}$} \\
\hline $72 \times 72 \times 1.2$ & 7,84 & 12,88 & 5,76 & 14,94 \\
\hline $72 \times 72 \times 1.5$ & 11,56 & 19,25 & 8,50 & 23,2 \\
\hline $72 \times 72 \times 1.9$ & 17,60 & 29,7 & 12,95 & 37,0 \\
\hline $92 \times 92 \times 1.2$ & 7,82 & 12,85 & 5,74 & 14,86 \\
\hline $92 \times 92 \times 1.5$ & 11,53 & 19,2 & 8,47 & 23,1 \\
\hline $92 \times 92 \times 1.9$ & 17,56 & 29,7 & 12,92 & 36,9 \\
\hline \multicolumn{5}{|l|}{$\mathrm{N}=100 \mathrm{~mm}$} \\
\hline $72 \times 72 \times 1.2$ & 10,85 & 16,62 & 7,46 & 15,71 \\
\hline $72 \times 72 \times 1.5$ & 14,82 & 23,2 & 10,63 & 24,1 \\
\hline $72 \times 72 \times 1.9$ & 21,26 & 34,3 & 15,65 & 38,2 \\
\hline $92 \times 92 \times 1.2$ & 10,81 & 16,58 & 7,43 & 15,63 \\
\hline $92 \times 92 \times 1.5$ & 14,78 & 23,1 & 10,59 & 24,0 \\
\hline $92 \times 92 \times 1.9$ & 21,22 & 34,3 & 15,61 & 38,1 \\
\hline \multicolumn{5}{|l|}{$\mathrm{N}=150 \mathrm{~mm}$} \\
\hline $72 \times 72 \times 1.2$ & 14,31 & 21,2 & 9,15 & 16,47 \\
\hline $72 \times 72 \times 1.5$ & 19,15 & 28,9 & 12,75 & 25,1 \\
\hline $72 \times 72 \times 1.9$ & 26,4 & 40,6 & 18,35 & 39,4 \\
\hline $92 \times 92 \times 1.2$ & 14,26 & 21,1 & 9,12 & 16,39 \\
\hline $92 \times 92 \times 1.5$ & 19,11 & 28,8 & 12,71 & 25,0 \\
\hline $92 \times 92 \times 1.9$ & 26,3 & 40,5 & 18,30 & 39,3 \\
\hline \multicolumn{5}{|l|}{$\mathrm{N}=200 \mathrm{~mm}$} \\
\hline $72 \times 72 \times 1.2$ & 17,77 & 25,8 & 10,85 & 17,24 \\
\hline $72 \times 72 \times 1.5$ & 23,5 & 34,6 & 14,88 & 26,1 \\
\hline $72 \times 72 \times 1.9$ & 31,9 & 47,8 & 21,05 & 40,7 \\
\hline $92 \times 92 \times 1.2$ & 17,71 & 25,7 & 10,81 & 17,15 \\
\hline $92 \times 92 \times 1.5$ & 23,4 & 34,5 & 14,83 & 26,0 \\
\hline $92 \times 92 \times 1.9$ & 31,8 & 47,8 & 21,0 & 40,5 \\
\hline
\end{tabular}


TABLA \#7b Fuerza Crítica Nominal del Pandeo del Alma,

$P_{n}$, para los productos de TUBOTICO

\begin{tabular}{|c|c|c|c|c|}
\hline SECCION & $\begin{array}{c}\text { CASO A } \\
\mathrm{Pn} \\
(\mathrm{kN})\end{array}$ & $\begin{array}{c}\text { CASO B } \\
\mathrm{Pn} \\
(\mathrm{kN})\end{array}$ & $\begin{array}{c}\text { CASO C } \\
\mathrm{Pn} \\
(\mathrm{kN})\end{array}$ & $\begin{array}{c}\text { CASO D } \\
\mathrm{Pn} \\
(\mathrm{kN})\end{array}$ \\
\hline $\mathbf{N}=50 \mathrm{~mm}$ & \multicolumn{4}{|l|}{} \\
\hline $38 \times 38 \times 2.4$ & 26,6 & 45,3 & 19,6 & 58,0 \\
\hline $50 \times 50 \times 1.8$ & 16,0 & 26,9 & 11,8 & 33,4 \\
\hline $50 \times 50 \times 2.4$ & 26,5 & 45,2 & 19,5 & 57,9 \\
\hline $72 \times 72 \times 1.8$ & 16,0 & 26,9 & 11,8 & 33,2 \\
\hline $72 \times 72 \times 3.2$ & 45,0 & 77,7 & 33,2 & 102,0 \\
\hline $100 \times 100 \times 1.8$ & 15,9 & 26,8 & 11,7 & 33,1 \\
\hline $100 \times 100 \times 3.2$ & 45,0 & 77,6 & 33,1 & 101,8 \\
\hline $125 \times 125 \times 1.8$ & 15,9 & 26,8 & 11,7 & 32,9 \\
\hline $125 \times 125 \times 3.2$ & 44,9 & 77,5 & 33,0 & 101,5 \\
\hline $125 \times 125 \times 4.8$ & 96,4 & 168,5 & 71,0 & 227,3 \\
\hline $150 \times 150 \times 1.8$ & 15,8 & 26,7 & 11,6 & 32,8 \\
\hline $150 \times 150 \times 3.2$ & 44,8 & 77,4 & 33,0 & 101,3 \\
\hline $150 \times 150 \times 4.8$ & 96,4 & 168,4 & 70,9 & 227,0 \\
\hline $\mathbf{N}=100 \mathrm{~mm}$ & & & & \\
\hline $38 \times 38 \times 2.4$ & $31,2^{*}$ & $51,1^{*}$ & $23,0^{*}$ & $59,6^{*}$ \\
\hline $50 \times 50 \times 1.8$ & 19,5 & 31,3 & 14,3 & 34,5 \\
\hline $50 \times 50 \times 2.4$ & 31,1 & 51,0 & 22,9 & 59,5 \\
\hline $72 \times 72 \times 1.8$ & 19,4 & 31,2 & 14,3 & 34,4 \\
\hline $72 \times 72 \times 3.2$ & 51,2 & 85,4 & 37,7 & 104,1 \\
\hline $100 \times 100 \times 1.8$ & 19,4 & 31,2 & 14,3 & 34,2 \\
\hline $100 \times 100 \times 3.2$ & 51,1 & 85,3 & 37,6 & 103,8 \\
\hline $125 \times 125 \times 1.8$ & 19,3 & 31,1 & 14,2 & 34,1 \\
\hline $125 \times 125 \times 3.2$ & 51,0 & 85,2 & 37,5 & 103,6 \\
\hline $125 \times 125 \times 4.8$ & 105,6 & 180,1 & 77,8 & 230,4 \\
\hline $150 \times 150 \times 1.8$ & 19,3 & 31,1 & 14,2 & 33,9 \\
\hline $150 \times 150 \times 3.2$ & 50,9 & 85,1 & 37,5 & 103,3 \\
\hline $150 \times 150 \times 4.8$ & 105,5 & 180,0 & 77,7 & 230,0 \\
\hline
\end{tabular}


Tala \#7b continuación

\begin{tabular}{|c|c|c|c|c|}
\hline SECCION & $\begin{array}{c}\text { CASO A } \\
\text { Pn } \\
(\mathrm{kN})\end{array}$ & $\begin{array}{c}\text { CASO B } \\
\text { Pn } \\
(\mathrm{kN})\end{array}$ & $\begin{array}{c}\text { CASO C } \\
\text { Pn } \\
(\mathrm{kN})\end{array}$ & $\begin{array}{c}\text { CASO D } \\
\text { Pn } \\
(k N)\end{array}$ \\
\hline \multicolumn{5}{|l|}{$\mathrm{N}=150 \mathrm{~mm}$} \\
\hline $38 \times 38 \times 2.4$ & $36,3^{*}$ & $57^{*}$ & $26,4^{*}$ & $61,1^{*}$ \\
\hline $50 \times 50 \times 1.8$ & $24,6^{*}$ & $37,6^{*}$ & $16,9^{*}$ & $35,7^{*}$ \\
\hline $50 \times 50 \times 2.4$ & $36,3^{*}$ & $56,9^{*}$ & $26,3^{*}$ & $61,1^{*}$ \\
\hline $72 \times 72 \times 1.8$ & 24,5 & 37,5 & 16,9 & 35,5 \\
\hline $72 \times 72 \times 3.2$ & 55,2 & 88,9 & 42,2 & 106,1 \\
\hline $100 \times 100 \times 1.8$ & 24,4 & 37,4 & 16,8 & 35,4 \\
\hline $100 \times 100 \times 3.2$ & 57,2 & 93,0 & 42,1 & 105,9 \\
\hline $125 \times 125 \times 1.8$ & 24,4 & 37,3 & 16,7 & 35,2 \\
\hline $125 \times 125 \times 3.2$ & 57,1 & 92,9 & 42,0 & 105,6 \\
\hline $125 \times 125 \times 4.8$ & 114,8 & 191,7 & 84,6 & 233,5 \\
\hline $150 \times 150 \times 1.8$ & 24,3 & 37,3 & 16,7 & 35,1 \\
\hline $150 \times 150 \times 3.2$ & 57,0 & 92,8 & 42,0 & 105,4 \\
\hline $150 \times 150 \times 4.8$ & 114,7 & 191,5 & 84,4 & 233,1 \\
\hline \multicolumn{5}{|l|}{\begin{tabular}{|l|}
$\mathrm{N}=200 \mathrm{~mm}$ \\
\end{tabular}} \\
\hline $38 \times 38 \times 2.4$ & $43,3^{*}$ & $66,1^{*}$ & $29,8^{*}$ & $62,6^{*}$ \\
\hline $50 \times 50 \times 1.8$ & $29,8^{*}$ & $44,4^{*}$ & $19,5^{*}$ & $36,8^{*}$ \\
\hline $50 \times 50 \times 2.4$ & $43,2^{*}$ & $66,0^{*}$ & $29,7^{*}$ & $62,5^{*}$ \\
\hline $72 \times 72 \times 1.8$ & 29,7 & 44,4 & 19,4 & 36,7 \\
\hline $72 \times 72 \times 3.2$ & $64,4^{*}$ & $101,0^{*}$ & $46,7^{*}$ & $108,2^{*}$ \\
\hline $100 \times 100 \times 1.8$ & 29,6 & 44,3 & 19,3 & 36,5 \\
\hline $100 \times 100 \times 3.2$ & 64,3 & 100,9 & 46,6 & 107,9 \\
\hline $125 \times 125 \times 1.8$ & 29,5 & 44,2 & 19,3 & 36,4 \\
\hline $125 \times 125 \times 3.2$ & 64,2 & 100,8 & 46,5 & 107,6 \\
\hline $125 \times 125 \times 4.8$ & 124,0 & 203,2 & 91,3 & 236,5 \\
\hline $150 \times 150 \times 1.8$ & 29,5 & 44,1 & 19,2 & 36,2 \\
\hline $150 \times 150 \times 3.2$ & 64,1 & 100,7 & 46,4 & 107,4 \\
\hline $150 \times 150 \times 4.8$ & 123,9 & 203,1 & 91,2 & 236,2 \\
\hline
\end{tabular}

Donde:

$P_{n}=$ Fuerza Crítica Nominal del Pandeo del Alma. Debe modificarse con el Factor de Seguridad o con el Factor de Resistencia;

- usando el Método de Esfuerzos de Trabajo (ASD) se divide entre el Factor de Seguridad: $\Omega_{\mathrm{w}}=1,85$.

- usando el Método de Esfuerzos Últimos (LRFD) se multiplica por el Factor de Resistencia: $\Phi_{\mathrm{W}}=0,75$.

$N=$ Ancho del Apoyo (aplastamiento).
CASO A: Reúne las reacciones o cargas concentradas en los extremos de una viga o voladizo cuando la distancia entre el borde del apoyo de la fuerza y el borde de la viga es menor de $1,5 h$ y cuando la separación entre los bordes de los apoyos de las cargas o reacciones adyacentes y opuestas, actuando éstas sea en el ala superior o inferior, es mayor de $1,5 \mathrm{~h}$.

CASO B: Reúne las reacciones o cargas concentradas cuando la distancia entre el borde del apoyo y el borde de la viga es igual o mayor de $1,5 h$ y cuando la separación entre los bordes de 
los apoyos de las cargas o reacciones adyacentes y opuestas, actuando éstas sea en el ala superior o inferior, es mayor de $1,5 h$.

CASO C: Incluye las reacciones o cargas concentradas en los extremos de una viga o voladizo cuando la distancia entre el borde del apoyo de la fuerza y el borde de la viga es menor de 1,5h y cuando la separación entre los bordes de los apoyos de las cargas o reacciones adyacentes y opuestas, actuando éstas simultáneamente en el ala superior e inferior, es igual o menor de 1,5h.
CASO D: Incluye las reacciones o cargas concentradas cuando la distancia entre el borde del apoyo y el borde de la viga es igual o mayor de $1,5 h$ y cuando la separación entre los bordes de los apoyos de las cargas o reacciones adyacentes y opuestas, actuando éstas simultáneamente en el ala superior e inferior, es igual o menor de $1,5 \mathrm{~h}$.

Donde $h$ es la altura de la parte plana del alma.

En la Figura 2 se muestran gráficamente las condiciones de los cuatro casos.
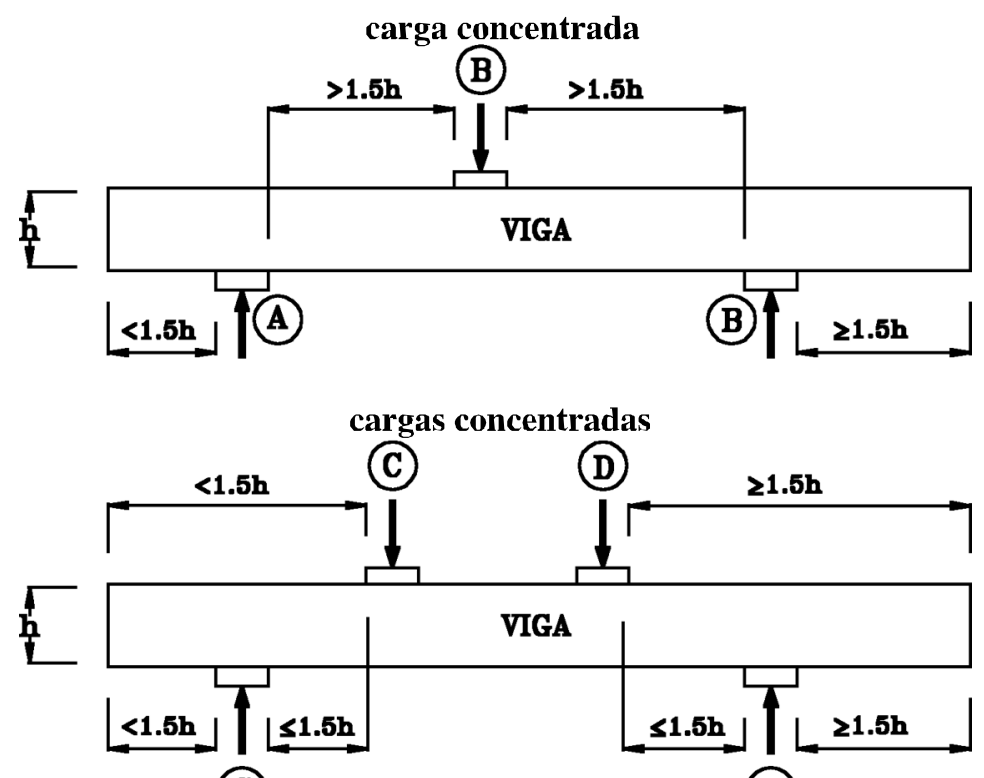

(C)

(D)

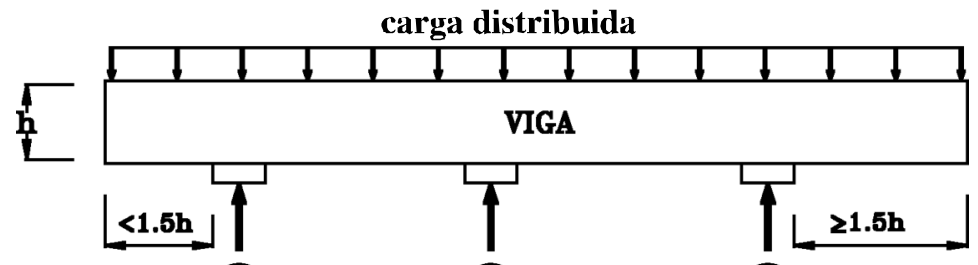

(A) (B)

Figura \#2 Esquemas de las diferentes condiciones de las cargas concentradas 
CAPACIDADES DE LOS TUBOS ESTRUCTURALES COMO COLUMNAS

Según la Sección C4 de AISI, la fuerza axial nominal $P_{n}$ en un elemento comprimido céntricamente será: $P_{n}=A_{e} F_{n}$

Donde:

$A_{e}=$ área efectiva de la sección de un elemento céntricamente comprimido con los esfuerzos $F_{n}$ y que depende de estos esfuerzos.
Usando el Método de Esfuerzos de Trabajo, (ASD), la fuerza axial nominal Pn debe dividirse entre el Factor de Seguridad $\Omega_{\mathrm{c}}=$ 1.80 y usando el Método de Esfuerzos Últimos (LRFD) debe multiplicarse por el Factor de resistencia $\Phi_{\mathrm{c}}=0,85$.

\section{COLUMNAS RESTRINGIDAS AL PANDEO}

En las columnas restringidas al pandeo, $P_{n}$ depende únicamente del área efectiva de la sección del perfil y ésta a su vez de los esfuerzos $f$. Las Tablas 8 muestran la variación del área efectiva, $A_{e}$, en función de los esfuerzos $f$.

Tabla \#8a Área Efectiva en función de Esfuerzo f en $\mathrm{cm}^{2}$ para los productos de Metalco

\begin{tabular}{|c|c|c|c|c|c|c|}
\hline$f$ & $72 \times 72 \times 1.2$ & $72 \times 72 \times 1.5$ & $72 \times 72 \times 1.9$ & $92 \times 92 \times 1.2$ & $92 \times 92 \times 1.5$ & $92 \times 92 \times 1.9$ \\
\hline $\mathrm{MPa}$ & & & & & & \\
\hline 227 & 2,75 & 3,94 & \multirow{4}{*}{ 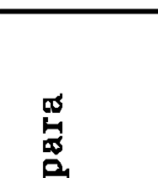 } & 2,92 & 4,31 & 6,35 \\
\hline 220 & 2,77 & 3,98 & & 2,96 & 4,35 & 6,41 \\
\hline 210 & 2,81 & 4,02 & & 3,01 & 4,42 & 6,48 \\
\hline 200 & 2,86 & 4,07 & & 3,06 & 4,49 & 6,56 \\
\hline 190 & 2,90 & 4,12 & \multirow{4}{*}{ 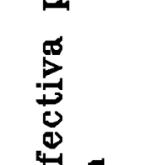 } & 3,12 & 4,56 & 6,65 \\
\hline 180 & 2,95 & 4,13 & & 3,18 & 4,64 & 6,69 \\
\hline 170 & 3,00 & 4,13 & & 3,24 & 4,72 & 6,69 \\
\hline 160 & 3,05 & 4,13 & & 3,31 & 4,80 & 6,69 \\
\hline 150 & 3,11 & 4,13 & \multirow{2}{*}{ Ð } & 3,38 & 4,89 & 6,69 \\
\hline 140 & 3,17 & 4,13 & & 3,46 & 4,99 & 6,69 \\
\hline 130 & 3,23 & 4,13 & \multirow{3}{*}{ 㤩 } & 3,54 & 5,09 & 6,69 \\
\hline 120 & 3,30 & 4,13 & & 3,64 & 5,20 & 6,69 \\
\hline 110 & 3,34 & 4,13 & & 3,74 & 5,31 & 6,69 \\
\hline 100 & 3,34 & 4,13 & \multirow{2}{*}{$\stackrel{\vec{D}}{\vec{D}}$} & 3,85 & 5,33 & 6,69 \\
\hline 90 & 3,34 & 4,13 & & 3,97 & 5,33 & 6,69 \\
\hline 80 & 3,34 & 4,13 & 目 & 4,10 & 5,33 & 6,69 \\
\hline 70 & 3,34 & 4,13 & & 4,25 & 5,33 & 6,69 \\
\hline 60 & 3,34 & 4,13 & \multirow{6}{*}{$\begin{array}{l}B^{4} \\
-0 \\
⿱ 0 \\
D \\
D \\
\forall\end{array}$} & 4,30 & 5,33 & 6,69 \\
\hline 50 & 3,34 & 4,13 & & 4,30 & 5,33 & 6,69 \\
\hline 40 & 3,34 & 4,13 & & 4,30 & 5,33 & 6,69 \\
\hline 30 & 3,34 & 4,13 & & 4,30 & 5,33 & 6,69 \\
\hline 20 & 3,34 & 4,13 & & 4,30 & 5,33 & 6,69 \\
\hline 10 & 3,34 & 4,13 & & 4,30 & 5,33 & 6,69 \\
\hline
\end{tabular}


Tabla \#8b Área Efectiva en función de Esfuerzo f en $\mathrm{cm}^{2}$ para los productos de TUBOTICO

\begin{tabular}{|c|c|c|c|c|c|c|c|}
\hline f & \multirow[t]{2}{*}{$38 \times 38 \times 2.4$} & \multirow[t]{2}{*}{$50 \times 50 \times 1.8$} & \multirow[t]{2}{*}{$50 \times 50 \times 2.4$} & \multirow[t]{2}{*}{$72 \times 72 \times 1.8$} & \multirow[t]{2}{*}{$72 \times 72 \times 3.2$} & \multirow[t]{2}{*}{$100 \times 100 \times 1.8$} & \multirow[t]{2}{*}{$100 \times 100 \times 3.2$} \\
\hline $\mathrm{MPa}$ & & & & & & & \\
\hline 227 & \multirow{5}{*}{ 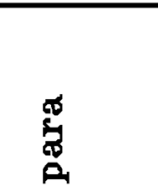 } & \multirow{5}{*}{ 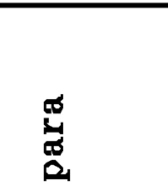 } & \multirow{5}{*}{ 芯 } & \multirow{4}{*}{ 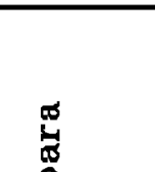 } & \multirow{4}{*}{ జ } & 6,02 & \multirow{4}{*}{ 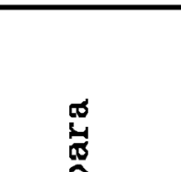 } \\
\hline 220 & & & & & & 6,07 & \\
\hline 210 & & & & & & 6,16 & \\
\hline 200 & & & & & & 6,25 & \\
\hline 190 & & & & \multirow{4}{*}{ 哯 } & \multirow{4}{*}{ 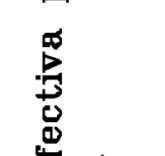 } & 6,34 & \multirow{4}{*}{$\underbrace{\infty}_{0}$} \\
\hline 180 & \multirow{4}{*}{ 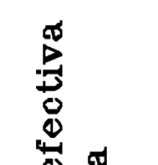 } & \multirow{3}{*}{ 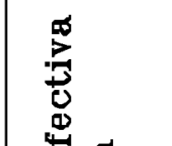 } & \multirow{3}{*}{ 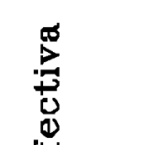 } & & & 6,44 & \\
\hline 170 & & & & & & 6,54 & \\
\hline 160 & & & & & & 6,64 & \\
\hline 150 & & \multirow{5}{*}{ 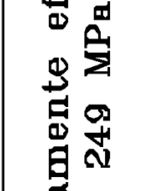 } & \multirow{5}{*}{ 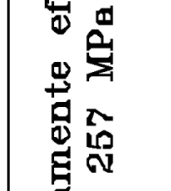 } & \multirow{5}{*}{ 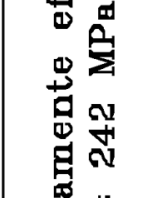 } & \multirow{5}{*}{ 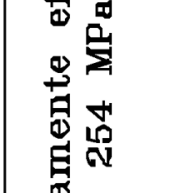 } & 6,76 & \multirow{5}{*}{ 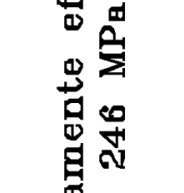 } \\
\hline 140 & \multirow{2}{*}{$\stackrel{\Delta}{\Sigma}$} & & & & & 6,87 & \\
\hline 130 & & & & & & 6,93 & \\
\hline 120 & مع & & & & & 6,93 & \\
\hline 110 & \multirow{2}{*}{ 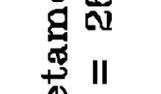 } & & & & & 6,93 & \\
\hline 100 & & \multirow{2}{*}{ 跑 } & \multirow{2}{*}{ 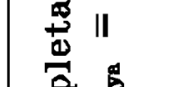 } & \multirow{2}{*}{ 总 } & \multirow{2}{*}{ 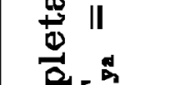 } & 6,93 & \multirow{2}{*}{ 营 } \\
\hline 90 & \multirow{4}{*}{$\begin{array}{l}\stackrel{0}{\theta_{1}} \\
0_{0}^{A}\end{array}$} & & & & & 6,93 & \\
\hline 80 & & \multirow{3}{*}{ 。 } & 当乎 & 宫的 & ⿷匚 & 6,93 & 它 \\
\hline 70 & & & 0 & 总 II & 8 & 6,93 & 量 II \\
\hline 60 & & & 4 & 0 & 4 & 6,93 & \\
\hline 50 & & E & 臬 & 品 & 8 & 6,93 & 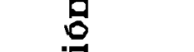 \\
\hline 40 & 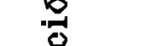 & 光 & 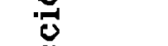 & 0 & 苑 & 6,93 & 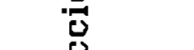 \\
\hline 30 & O & 巳 & d & 导 & 导 & 6,93 & 0 \\
\hline 20 & 岁 & & & & & 6,93 & \\
\hline 10 & & & & & & 6,93 & \\
\hline
\end{tabular}


Tabla \#8b continuación

\begin{tabular}{|c|c|c|c|c|c|c|}
\hline$f$ & \multirow[t]{2}{*}{$125 \times 125 \times 1.8$} & \multirow[t]{2}{*}{$125 \times 125 \times 3.2$} & \multirow[t]{2}{*}{$125 \times 125 \times 4.8$} & \multirow[t]{2}{*}{$150 \times 150 \times 1.8$} & \multirow[t]{2}{*}{$150 \times 150 \times 3.2$} & \multirow[t]{2}{*}{$150 \times 150 \times 4.8$} \\
\hline $\mathrm{MPa}$ & & & & & & \\
\hline 227 & 6,42 & \multirow{5}{*}{ 苂 } & \multirow{4}{*}{ జ్ర } & 6,69 & 17,52 & \multirow{5}{*}{ 总 } \\
\hline 220 & 6,49 & & & 6,77 & 17,66 & \\
\hline 210 & 6,59 & & & 6,89 & 17,87 & \\
\hline 200 & 6,71 & & & 7,01 & 18,08 & \\
\hline 190 & 6,82 & & \multirow{4}{*}{$\underbrace{\infty}_{0}$} & 7,15 & 18,19 & \\
\hline 180 & 6,95 & \multirow{5}{*}{ 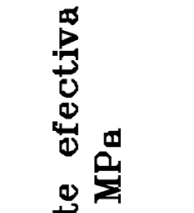 } & & 7,29 & 18,19 & \multirow{3}{*}{$\underset{0}{\infty}$} \\
\hline 170 & 7,08 & & & 7,44 & 18,19 & \\
\hline 160 & 7,22 & & & 7,60 & 18,19 & \\
\hline 150 & 7,37 & & \multirow{5}{*}{ 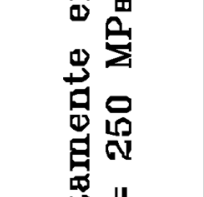 } & 7,78 & 18,19 & \multirow{5}{*}{ 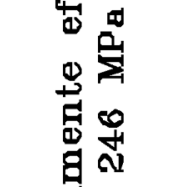 } \\
\hline 140 & 7,53 & & & 7,97 & 18,19 & \\
\hline 130 & 7,70 & \multirow{3}{*}{ 丞 } & & 8,17 & 18,19 & \\
\hline 120 & 7,89 & & & 8,40 & 18,19 & \\
\hline 110 & 8,09 & & & 8,64 & 18,19 & \\
\hline 100 & 8,31 & \multirow{4}{*}{ 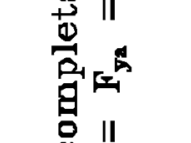 } & \multirow{4}{*}{ 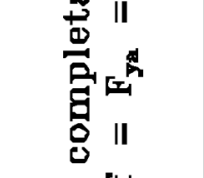 } & 8,91 & 18,19 & \multirow{4}{*}{ 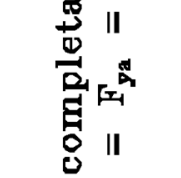 } \\
\hline 90 & 8,54 & & & 9,21 & 18,19 & \\
\hline 80 & 8,73 & & & 9,55 & 18,19 & \\
\hline 70 & 8,73 & & & 9,92 & 18,19 & \\
\hline 60 & 8,73 & 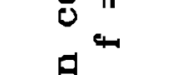 & \multirow{6}{*}{ 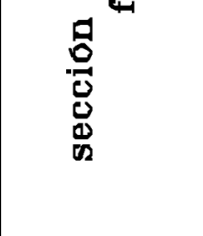 } & 10,34 & 18,19 & \multirow{5}{*}{$\begin{array}{l}a^{4} \\
0 \\
0 \\
0 \\
0 \\
0 \\
0\end{array}$} \\
\hline 50 & 8,73 & \multirow{5}{*}{ 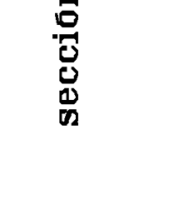 } & & 10,53 & 18,19 & \\
\hline 40 & 8,73 & & & 10,53 & 18,19 & \\
\hline 30 & 8,73 & & & 10,53 & 18,19 & \\
\hline 20 & 8,73 & & & 10,53 & 18,19 & \\
\hline 10 & 8,73 & & & 10,53 & 18,19 & \\
\hline
\end{tabular}




\section{COLUMNAS CON POSIBILIDAD DEL PANDEO}

En las columnas con posibilidad del pandeo, $P_{n}$ depende del área efectiva y de las longitudes del pandeo $K L$. Las Tablas 9 muestran los valores de $P_{n}$ para las diferentes longitudes del pandeo considerando el área efectiva.

Tabla \#9a Fuerza Axial Nominal Concentrada $P_{n}$ en $k N$ para los productos de METALCO

\begin{tabular}{|c|c|c|c|c|c|c||}
\hline $\mathrm{KL}$ & $\mathbf{7 2 \times 7 2 \times 1 . 2}$ & $\mathbf{7 2 \times 7 2 \times 1 . 5}$ & $\mathbf{7 2 \times 7 2 \times 1 . 9}$ & $\mathbf{9 2 \times 9 2 \times 1 . 2}$ & $\mathbf{9 2 \times 9 2 \times 1 . 5}$ & $\mathbf{9 2 \times 9 2 \times 1 . 9}$ \\
\hline $\mathrm{m}$ & & & & & & \\
\hline 0,25 & 62,2 & 89,3 & 125,1 & 66,3 & 97,7 & 144,0 \\
\hline 0,50 & 61,7 & 88,6 & 123,7 & 66,0 & 97,3 & 143,3 \\
\hline 0,75 & 61,0 & 87,4 & 121,3 & 65,6 & 96,6 & 142,1 \\
\hline 1,00 & 59,9 & 85,7 & 118,0 & 64,9 & 95,6 & 140,5 \\
\hline 1,25 & 58,6 & 83,7 & 113,9 & 64,1 & 94,3 & 138,4 \\
\hline 1,50 & 57,1 & 81,2 & 109,0 & 63,1 & 92,8 & 135,9 \\
\hline 1,75 & 55,3 & 78,3 & 103,6 & 62,0 & 91,0 & 133,0 \\
\hline 2,00 & 53,2 & 74,3 & 97,7 & 60,7 & 89,0 & 129,7 \\
\hline 2,25 & 51,0 & 69,9 & 91,4 & 59,3 & 86,7 & 126,0 \\
\hline 2,50 & 48,6 & 65,3 & 84,8 & 57,7 & 84,2 & 121,6 \\
\hline 2,75 & 46,0 & 60,5 & 78,1 & 56,0 & 81,6 & 116,0 \\
\hline 3,00 & 43,4 & 55,6 & 71,3 & 54,2 & 78,8 & 110,2 \\
\hline 3,25 & 40,6 & 50,8 & 64,6 & 52,3 & 75,8 & 104,3 \\
\hline 3,50 & 37,6 & 46,1 & 58,1 & 50,3 & 72,7 & 98,2 \\
\hline 3,75 & 33,9 & 41,5 & 51,8 & 48,2 & 69,4 & 92,1 \\
\hline 4,00 & 30,4 & 37,1 & 45,8 & 46,1 & 66,1 & 85,9 \\
\hline 4,25 & 26,9 & 32,9 & 40,6 & 43,9 & 62,6 & 79,8 \\
\hline 4,50 & 24,0 & 29,3 & 36,2 & 41,7 & 59,1 & 73,9 \\
\hline 4,75 & 21,6 & 26,3 & 32,5 & 39,4 & 54,9 & 68,0 \\
\hline 5,00 & 19,5 & 23,7 & 29,3 & 37,1 & 50,4 & 62,4 \\
\hline 5,25 & 17,7 & 21,5 & 26,6 & 34,8 & 46,0 & 56,8 \\
\hline 5,50 & 16,1 & 19,6 & 24,2 & 32,5 & 41,9 & 51,8 \\
\hline 5,75 & 14,7 & 18,0 & 22,2 & 30,5 & 38,4 & 47,4 \\
\hline 6,00 & 13,5 & 16,5 & 20,4 & 28,6 & 35,2 & 43,5 \\
\hline & & & & & & \\
\hline
\end{tabular}


Tabla \#9b Fuerza Axial Nominal Concentrada $P_{n}$ en kN para los productos de TUBOTICO

\begin{tabular}{|c|c|c|c|c|c|c|c|c|c|c|c|c|c|c|c|c|c|c|c|c|c|c|c|c|c|c|}
\hline 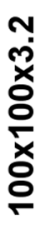 & & ন্ & 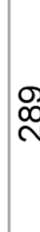 & $\stackrel{\complement}{a}$ & 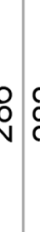 & 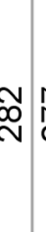 & & $\stackrel{0}{\stackrel{N}{N}}$ & $\stackrel{\mathscr{N}}{\mathscr{N}}$ & $\stackrel{\llcorner}{\stackrel{\circ}{N}}$ & $\stackrel{Q}{\sim}$ & $\stackrel{\mathscr{N}}{\mathcal{N}}$ & $\stackrel{\mathscr{N}}{N}$ & $\frac{n}{\sim}$ & ত্ & $\begin{array}{l}N \\
\tilde{N} \\
\stackrel{9}{\sigma}\end{array}$ & $\begin{array}{c}\infty \\
\overleftarrow{\infty} \\
\leftarrow\end{array}$ & $\begin{array}{l}m \\
\rho^{\prime} \\
\end{array}$ & $\begin{array}{l}0 \\
\infty \\
\infty \\
\infty \\
\sigma\end{array}$ & 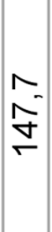 & $\begin{array}{l}0 \\
\mathscr{0} \\
\stackrel{-}{\sigma}\end{array}$ & $\begin{array}{l}\sigma \\
1 \tilde{L}^{-} \\
\stackrel{\sim}{\sim}\end{array}$ & & 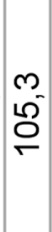 & $\begin{array}{l}\nabla \\
\mathscr{6}\end{array}$ & \\
\hline 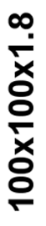 & & $\begin{array}{l}\vec{\nabla} \\
\stackrel{0}{ } \\
\stackrel{-}{ }\end{array}$ & $\begin{array}{l}\infty \\
1 \\
m \\
\sim\end{array}$ & 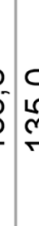 & $\begin{array}{l}2 \\
2 \\
2 \\
2\end{array}$ & $\vec{m}$ & $\begin{array}{l}\stackrel{N}{\sim} \\
\stackrel{2}{\leftarrow}\end{array}$ & $\begin{array}{l}m \\
\stackrel{0}{\circ} \\
\stackrel{m}{\sigma}\end{array}$ & $\stackrel{\check{\infty}}{\stackrel{5}{\sim}}$ & 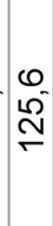 & $\stackrel{\infty}{N}$ & $\frac{\sqrt{\sigma}}{\bar{\sigma}}$ & $\stackrel{+}{\oplus}$ & 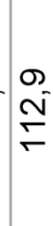 & 믐 & $\stackrel{5}{\circ}$ & $\frac{0}{5}$ & $\hat{\mathscr{O}}$ & क & ळ́ & & $\begin{array}{l}N^{-} \\
N^{\prime}\end{array}$ & & $\begin{array}{l}0 \\
\tilde{\mathscr{C}}\end{array}$ & $\begin{array}{l}0 \\
\infty \\
\infty \\
10\end{array}$ & $\begin{array}{l}\text { क } \\
\text { న్ }\end{array}$ \\
\hline 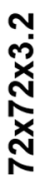 & & 은 & $\grave{尺}$ & ণ & $\vec{s}$ & $\overrightarrow{8}$ & $\begin{array}{l}\check{\infty} \\
\infty \\
\infty\end{array}$ & $\begin{array}{l}\checkmark \\
\delta^{\circ} \\
\infty\end{array}$ & $\begin{array}{l}0 \\
0 \\
0 \\
N\end{array}$ & 冓 & $\begin{array}{l}0 \\
\stackrel{\infty}{+}\end{array}$ & $\begin{array}{l}\infty \\
\stackrel{\infty}{\sigma} \\
\stackrel{-}{-}\end{array}$ & 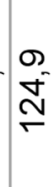 & $\frac{\bar{\sigma}}{\Gamma}$ & م & \% & $\begin{array}{l}0 \\
\sigma^{\prime}\end{array}$ & $\begin{array}{l}\text { の } \\
\text { ఠ }\end{array}$ & $\begin{array}{c}\sigma \\
\sigma \\
\sigma\end{array}$ & $\begin{array}{l}N \\
10 \\
10\end{array}$ & 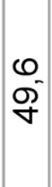 & 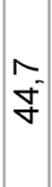 & & $\begin{array}{c}0 \\
\text { m. } \\
\text { na }\end{array}$ & $\begin{array}{l}\infty \\
\tilde{m}^{-}\end{array}$ & $\bar{m}$ \\
\hline $\begin{array}{l}\infty \\
\underset{x}{x} \\
\underset{N}{x} \\
\underset{N}{N}\end{array}$ & & \begin{tabular}{l}
$\infty^{0}$ \\
$\infty^{\circ}$ \\
\hdashline
\end{tabular} & $\frac{7}{7}$ & $=\begin{array}{l}a \\
5 \\
5\end{array}$ & $\begin{array}{l}0 \\
\\
-\end{array}$ & $\bar{F}$ & $\begin{array}{l}\text { D. } \\
\text { 음 }\end{array}$ & $\stackrel{m}{m}$ & $\begin{array}{l}\stackrel{N}{\infty} \\
\infty \\
\infty\end{array}$ & ๙ি & $\stackrel{N}{0}$ & 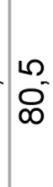 & $\check{r}^{-}$ & $\tilde{\sigma}$ & $\frac{\nabla}{\sigma}$ & $\begin{array}{l}m \\
0 \\
0 \\
\infty\end{array}$ & $\begin{array}{l}\dot{v} \\
\text { S্ }\end{array}$ & $\begin{array}{l}\mathscr{0} \\
\stackrel{\text { Pे }}{q}\end{array}$ & $\begin{array}{l}0 \\
\infty \\
\infty \\
m\end{array}$ & $\begin{array}{l}n \\
\text { mे }\end{array}$ & & 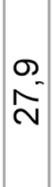 & & స్ & & $\begin{array}{l}\text { m } \\
\text { ल) } \\
\text { (") }\end{array}$ \\
\hline 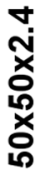 & & m & $\begin{array}{l}m \\
0 \\
0\end{array}$ & 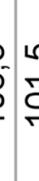 & 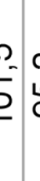 & م) & $\begin{array}{l}0 \\
N^{\prime}\end{array}$ & হ & $\infty^{m}$ & $\frac{N}{\sigma}$ & $\begin{array}{l}m \\
\text { N } \\
\text { no }\end{array}$ & $\begin{array}{l}0 \\
\text { f }\end{array}$ & $\begin{array}{l}\nabla \\
\stackrel{\Delta}{0}\end{array}$ & $\begin{array}{l}0 \\
- \\
0\end{array}$ & $\begin{array}{l}0 \\
0 \\
\stackrel{0}{*}\end{array}$ & $\begin{array}{l}\infty \\
\mathbb{N}\end{array}$ & $\begin{array}{l}0 \\
\stackrel{\sigma}{\square}\end{array}$ & $\begin{array}{l}\stackrel{0}{\check{N}} \\
\stackrel{-}{\sigma}\end{array}$ & $\begin{array}{l}\stackrel{2}{N} \\
\stackrel{2}{\sim}\end{array}$ & 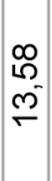 & & $\begin{array}{l}8 \\
\text { - } \\
\mp\end{array}$ & & $\begin{array}{l}\text { 8 } \\
\text { aे }\end{array}$ & & $\begin{array}{l}\text { ல } \\
\text { N }\end{array}$ \\
\hline 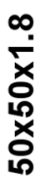 & & $\begin{array}{c}N \\
\infty \\
\infty\end{array}$ & 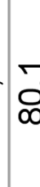 & & $\begin{array}{ll}0 \\
0 \\
0\end{array}$ & $\stackrel{n}{N}$ & $\begin{array}{l}0 \\
\mathscr{8}\end{array}$ & $\begin{array}{l}\infty \\
\delta^{-}\end{array}$ & $\begin{array}{l}\Delta \\
\dot{0}\end{array}$ & $\begin{array}{l}\infty \\
\text { f }\end{array}$ & $\frac{m}{\sigma}$ & $\overline{\check{m}}$ & $\begin{array}{l}\mathcal{N} \\
\mathbb{N}\end{array}$ & $\begin{array}{l}0 \\
\stackrel{+}{N}\end{array}$ & D & $\mid \begin{array}{l} \pm \\
\infty \\
\infty\end{array}$ & $\begin{array}{l}5 \\
\stackrel{N}{2}\end{array}$ & $\begin{array}{l}\check{\infty} \\
\mathcal{m}^{-}\end{array}$ & & $\begin{array}{l}\sigma \\
\sigma \\
0 \\
\sigma\end{array}$ & & $\begin{array}{l}\$ \\
\infty \\
\infty\end{array}$ & & $\begin{array}{l}\bar{m} \\
N\end{array}$ & & $\frac{ \pm}{6}$ \\
\hline 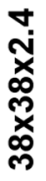 & & $\begin{array}{l}0 \\
\infty \\
\infty\end{array}$ & $\begin{array}{l}\checkmark \\
\infty \\
1\end{array}$ & $\left\{\begin{array}{l}0 \\
1 \\
1\end{array}\right.$ & $=8$ & $\widehat{\widetilde{0}}$ & $\begin{array}{l}0 \\
\mathbb{L}^{\circ}\end{array}$ & $\begin{array}{l}0 \\
\operatorname{lo}^{-}\end{array}$ & ח & $\begin{array}{l}\infty \\
N\end{array}$ & $\frac{\pi}{\bar{N}}$ & $\stackrel{N}{N}$ & 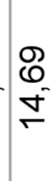 & 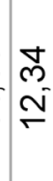 & $\begin{array}{l}5 \\
0 \\
0 \\
0\end{array}$ & Ó & $\begin{array}{l}8 \\
\stackrel{8}{-}\end{array}$ & $\begin{array}{l}\text { ठ } \\
\sigma^{-}\end{array}$ & & 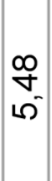 & & $\begin{array}{l}\text { \& } \\
\text { オ̇ }\end{array}$ & & $\begin{array}{l}\hat{0} \\
m^{-}\end{array}$ & & $\begin{array}{l}\text { व } \\
\text { m. }\end{array}$ \\
\hline$\underline{\underline{ }}$ & $E$ & $\mid \begin{array}{l}n \\
N \\
0\end{array}$ & in & 5 & 5 & $\begin{array}{l}8 \\
8 \\
5\end{array}$ & $\stackrel{\sim}{\stackrel{2}{r}}$ & 음 & $\stackrel{n}{\stackrel{2}{r}}$ & $\begin{array}{l}\mathrm{N} \\
\mathrm{N}\end{array}$ & $\stackrel{\sim}{N}$ & 足 & $\stackrel{n}{\stackrel{n}{N}}$ & 8 & {$\left[\begin{array}{l}n \\
N \\
m\end{array}\right.$} & $\begin{array}{l}0 \\
0 \\
m\end{array}$ & $\begin{array}{l}\infty \\
N \\
M^{-}\end{array}$ & $\begin{array}{l}8 \\
\text { ष }\end{array}$ & $\begin{array}{l}\stackrel{\Omega}{N} \\
\forall \\
\forall\end{array}$ & $\begin{array}{l}0 \\
\infty \\
\forall \\
\forall\end{array}$ & $\begin{array}{l}2 \\
\stackrel{2}{\sigma} \\
\forall\end{array}$ & $\begin{array}{l}8 \\
0 \\
15\end{array}$ & & $\begin{array}{l}0 \\
10 \\
10\end{array}$ & $\begin{array}{l}n \\
10 \\
10\end{array}$ & $\begin{array}{l}8 \\
6\end{array}$ \\
\hline
\end{tabular}




\begin{tabular}{|c|c|c|c|c|c|c|c|c|c|c|c|c|c|c|c|c|c|c|c|c|c|}
\hline 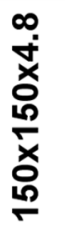 & & 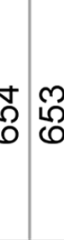 & 웅 & 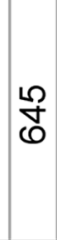 & 워 & & 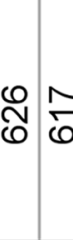 & & & $\begin{array}{l}\infty \\
\infty \\
\infty \\
0\end{array}$ & $\stackrel{m}{n}$ & $\mathbb{8}$ & 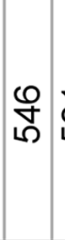 & $\overline{\mathscr{S}}$ & & Ş & \begin{tabular}{l}
$\infty$ \\
\hdashline \\
$\sigma$
\end{tabular} & זु & 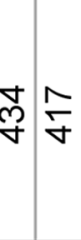 & ষ্ণ & $\begin{array}{l}m \\
\infty \\
\infty \\
m\end{array}$ \\
\hline 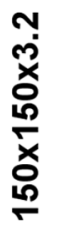 & & ক্ল & ঙ̊ల్ల & హ్ల & న్లి & $\begin{array}{l}\mathscr{\infty} \\
\stackrel{m}{\infty}\end{array}$ & 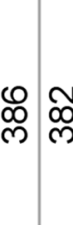 & 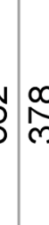 & o & $\begin{array}{l}8 \\
0\end{array}$ & 㣽 & $\begin{array}{l}\infty \\
\infty \\
\end{array}$ & 总 & ঙิ & 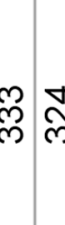 & 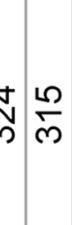 & ஜొ & 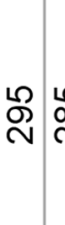 & 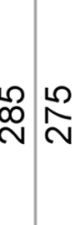 & $\begin{array}{l}0 \\
\varrho \\
\sim\end{array}$ & $\stackrel{\llcorner}{\stackrel{2}{N}}$ \\
\hline 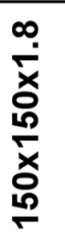 & & 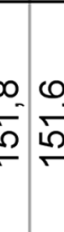 & & & $\begin{array}{l}0 \\
0 \\
0 \\
-1\end{array}$ & 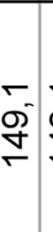 & 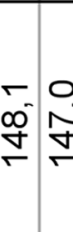 & & 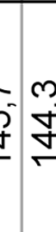 & 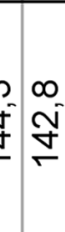 & $\frac{\pi}{\check{z}}$ & $\begin{array}{l}m \\
\stackrel{m}{m} \\
\stackrel{m}{m}\end{array}$ & $\begin{array}{c}m \\
\stackrel{m}{c} \\
\sigma\end{array}$ & $\begin{array}{c}m \\
\stackrel{m}{m} \\
\stackrel{m}{\longrightarrow}\end{array}$ & 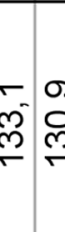 & 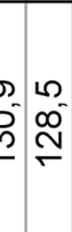 & $\begin{array}{l}0 \\
\stackrel{0}{-} \\
\stackrel{2}{N}\end{array}$ & 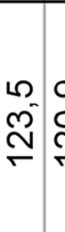 & 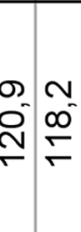 & $\begin{array}{l}\nabla \\
\mathscr{L}^{\prime} \\
\leftarrow\end{array}$ & $\stackrel{20}{\stackrel{2}{\mp}}$ \\
\hline 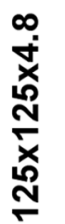 & & \begin{tabular}{l} 
Uి \\
\hdashline
\end{tabular} & & חొ & 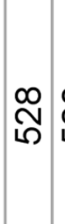 & ్ㅠ & $\underset{5}{5}$ & & & $\underset{f}{\tilde{f}} \tilde{\sigma}$ & 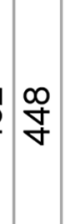 & ָे & $\frac{\mathscr{O}}{\dot{\gamma}}$ & ৪) & 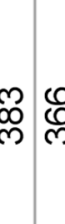 & : & $\bar{m}$ & $\frac{m}{m}$ & 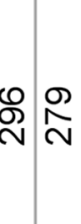 & $\underset{\mathrm{N}}{\mathrm{N}}$ & 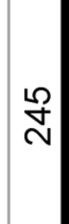 \\
\hline 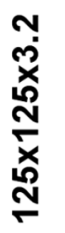 & & :ֶ) & & $\stackrel{\varphi}{\infty}$ & స్ల్ల & & ఫ্ল & & $\bar{j} \frac{\Omega}{m}$ & $\frac{\sigma}{\rho}$ & চ্ল & న్ & $\underset{\sim}{\infty}$ & 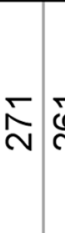 & 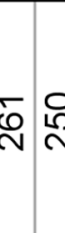 & 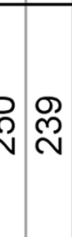 & $\widehat{\hat{N}}$ & $\stackrel{0}{N}$ & 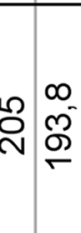 & $\begin{array}{l}\infty \\
i \\
\infty \\
\Gamma\end{array}$ & $\begin{array}{l}0 \\
\text { N } \\
0\end{array}$ \\
\hline 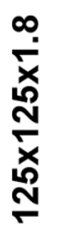 & & \begin{tabular}{lll}
0 & $m$ \\
$f^{-}$ & $n$ \\
\hdashline & $n$ \\
\hdashline
\end{tabular} & $\begin{array}{l}\checkmark \\
\\
\end{array}$ & 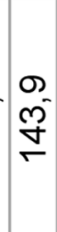 & : & & 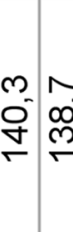 & & & 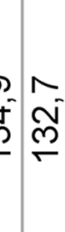 & 孞 & & 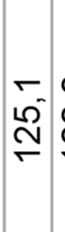 & $\begin{array}{l}m \\
\stackrel{N}{N}: \\
\stackrel{5}{?}\end{array}$ & & 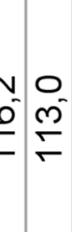 & $\stackrel{r}{g^{-}}$ & $\begin{array}{l}N \\
\mathscr{Q} \\
\underline{C}\end{array}$ & 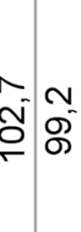 & $\begin{array}{l}0 \\
2 \\
0\end{array}$ & $\begin{array}{l}\infty \\
\bar{\sigma}\end{array}$ \\
\hline$\vec{x}$ & $E$ & 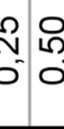 & & & $\stackrel{\stackrel{\Omega}{N}}{-}$ & $\stackrel{\circ}{\circ}$ & $\begin{array}{l}\stackrel{0}{=} \\
= \\
=\end{array}$ & & ป & 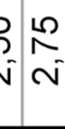 & ' & & $\begin{array}{l}\circ \\
0 \\
m \\
0\end{array}$ & $\begin{array}{l}\frac{n}{2} \\
m \\
m\end{array}$ & $\begin{array}{l}8 \\
\end{array}$ & 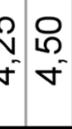 & $\begin{array}{l}\stackrel{L}{2} \\
\forall \\
\forall\end{array}$ & $\begin{array}{l}8 \\
8 \\
5 \\
5\end{array}$ & 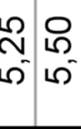 & $\begin{array}{l}n \\
0 \\
0^{\prime}\end{array}$ & $\begin{array}{l}8 \\
6 \\
0\end{array}$ \\
\hline
\end{tabular}


